\section{Assessment of behavioural toxicity in dichlorvos-exposed Caenorhabditis elegans}

\author{
Sarita Mishra, Rakhi Agarwal* \\ Laboratory of Analytical \& Molecular Toxicology (Forensic Chemistry \& Toxicology Laboratory), \\ School of Forensic Science, National Forensic Sciences University, Sector 09, Gandhinagar, 382007, \\ Gujarat, India
}

*Corresponding author, E-mail: lamt.dct.gfsu@gmail.com

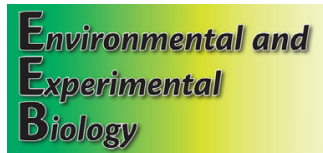

ISSN 2255-9582

UNIVERSITY OF LATVIA

\begin{abstract}
The extensive use of pesticides in agriculture and other household products have raised global health concerns due to their toxicity and adverse effects on humans and other non-target species. Dichlorvos (DDVP), an organophosphate pesticide, has become increasingly popular for its domestic, industrial and agricultural uses, however, it also raises a serious public health concern due to its unregulated and unrestricted use. Therefore, knowing the toxic nature of DDVP will be a great help to use it in a regulated manner. The present study was designed to examine the impact of DDVP exposure on animal behaviour, using a nematode species Caenorhabditis elegans. Exposure to C. elegans with varying concentrations of $\operatorname{DDVP}(5,20,45,70,90 \mu \mathrm{M})$ for $2 \mathrm{~h}$ induced a concentration-dependent reduction in feeding rate and altered locomotion in terms of speed, body bending, head thrashing, omega turn and reversal frequency. Thus, the present study suggested the behavioural toxicity associated with DDVP exposure and use of different behavioural parameters mentioned above as early biomarkers for toxicological studies using C. elegans.
\end{abstract}

Key words: behavioural toxicity, Caenorhabditis elegans, dichlorvos, early biomarkers, pharyngeal pumping. Abbreviations: DDVP, dichlorvos; NGM, Nematode growth medium; FR, feeding rate; RNAi, RNA interference.

\section{Introduction}

Pesticides are groups of heterogeneous chemicals used to control the pests, vector-borne and food-borne diseases, unwanted animals or plant species, interfering with production, processing, storage, transport or marketing of food (Weiss et al. 2004; Aktar et al. 2009). Pesticides include insecticides, fungicides, herbicides, disinfectants, plant growth regulators or any chemical meant for preventing defoliation or, premature fall of fruit (Levine 2007; Aktar et al. 2009). Although use of pesticides have provided immense help in controlling different pests (Aktar et al. 2009), at the same time the unregulated and unrestricted use of these chemicals have raised several health concerns and been referred to as a time bomb (Levine 2007; Gill Garg, 2014).

Dichlorvos (2,2-dichlorovinyl dimethyl phosphate; DDVP) is an organophosphate insecticide commonly used to control the household pests and to fumigate the crops (Shukla et al. 2017). It falls under Class 1B, i.e. a highly hazardous chemical (WHO 1992). It has been shown that DDVP inhibits acetylcholinesterase enzyme activity at neuromuscular junctions, causing overstimulation of neurons (Gupta 2006; Gupta et al. 2018), and it has negative impacts on human health (Okoroiwu, Iwara 2018). Being volatile, inhalation is the most common route of acute toxicity. It has been also shown that DDVP has acetylcholinesterase inhibitory activity in fish (Varo et al. 2003; Wang et al. 2004; Varo et al. 2007; 2008; Rao et al. 2017), acute toxicity in rats (Owoeye et al. 2012) and guinea pigs (Taylor et al.2008), oxidative injury and developmental toxicity in zebra fish (Sisman et al. 2010; Shukla et al. 2017), teratological effects on rabbits and rats (Thorpe et al. 1972), and ocular mitotic effects on rabbits and monkey (Ubels et al. 1987).

Here, we used Caenorhabditis elegans, a non-pathogenic soil nematode, which is commonly used to examine the toxicity associated with chemicals/toxicants and has been established as a useful model organism for the assessment of early biomarkers for toxicological screening (Hunt 2017). It is small in size $(\sim 1 \mathrm{~mm})$, has short life cycle (three days at $20^{\circ} \mathrm{C}$ ), high fecundity (300 to 1000 eggs) and low number of neurons ( 302; Apfeld et al. 2019). Due to the fully sequenced genome, with $\sim 20500$ genes located on six pairs of chromosomes, availability of RNAi mutants and other transgenic strains and $~ 40 \%$ similarity with human disease genes, make it as a model of choice for various studies (Apfeld et al. 2019). In recent years, C. elegans has been used as a model organism to study developmental, neuronal and behavioural toxicity (Hunt 2017; RaleySusman et al. 2018; Ruszkiewicz et al. 2018; Wittkowski et al. 2019). Study of behavioural toxicity in an animal model can be helpful to gain information about the toxic nature of a selected chemical/toxicant. Coordination of 
muscles and motor neurons in C. elegans involves a set of behaviours, such as locomotion, pharyngeal pumping, egg laying, reproductive, defecation, thermotaxis, chemotaxis, odortaxis, and mechanosensation behaviour (Hart 2006; Hobert 2013). The potential of C. elegans for toxicological assessment and high-throughput screening is well reported (Reilly et al. 2015; Schwendeman, Shaham 2016; Kinser, Pincus 2018; Teo et al. 2020) and measuring different behavioural parameters provides insights into a variety of molecular pathways and mechanisms (Perni et al. 2018). Behavioural phenotypic alterations can be an indicator of pathological conditions (Nawa, Matsuoka 2012) and may serve as early biomarkers for the onset of toxicity.

The aim of the present study was to explore the possible correlations of altered behavioural parameters i.e. pharyngeal pumping and locomotion in terms of head thrashing, body bend, speed, reversal frequency, and omega turn in C. elegans, induced by exposure to varying concentration of DDVP. Our study strongly suggested the use of different behavioural parameters in C. elegans as early biomarkers for the assessment of toxicity associated with various chemicals.

\section{Materials and methods}

\section{Chemicals and reagents used}

Agar, cholesterol, glucose, peptone, $\mathrm{KH}_{2} \mathrm{PO}_{4}, \mathrm{MgSO}_{4}$, $\mathrm{NH}_{4} \mathrm{Cl}, \mathrm{KOH}$ and uracil were procured from SRL chemicals (Mumbai, India). DDVP standard was procured from Cayman Chemical (USA). $\mathrm{NaCl}, \mathrm{CaCl}_{2}$, and $\mathrm{Na}_{2} \mathrm{HPO}_{4}$ were procured from Merck (USA). $\mathrm{NaClO}$ was procured from Fisher Scientific (United States). All chemicals and reagents used in the current study were highly pure (99.9\%).

\section{Model organism}

N2 strain (Wild type) of Caenorhabditis elegans was used in the current study. The worms were cultured on Nematode Growth Medium (NGM) plates containing $3 \mathrm{~g} \mathrm{~L}^{-1} \mathrm{NaCl}$, $2.5 \mathrm{~g} \mathrm{~L}^{-1}$ peptone, $17 \mathrm{~g} \mathrm{~L}^{-1}$ agar, $5 \mathrm{mg} \mathrm{L}^{-1}$ cholesterol, $1 \mathrm{~mL}$ $\mathrm{L}^{-1}$ of $1 \mathrm{M} \mathrm{CaCl}_{2}, 1 \mathrm{~mL} \mathrm{~L}^{-1}$ of $1 \mathrm{M} \mathrm{MgSO}_{4}, 25 \mathrm{~mL} \mathrm{~L}^{-1}$ of $1 \mathrm{M}$ $\mathrm{KH}_{2} \mathrm{PO}_{4}$ with pH 6.0, along with E. coli strain OP50 at $22^{\circ} \mathrm{C}$ (Brenner 1974; Stiernagle 2006).

The worm batch used in the current study was a generous gift from Dr. Aamir Nazir, Senior Scientist and Head, Fundamental Genomics and Molecular Toxicology Laboratory, Division of Toxicology, Council of Scientific and Industrial Research-Central Drug Research Institute (CSIR-CDRI), Lucknow, Uttar Pradesh, India.

The worms were exposed to DDVP $(5,20,45,70$ and 90 $\mu \mathrm{M})$ by selecting the synchronized L4 stage ( $\sim 60$ worms per well) for $2 \mathrm{~h}$ in 96 well plates at $22^{\circ} \mathrm{C}$.

Age synchronization of worms was done in order to avoid any variation due to the age difference of the worms. This was done by treating the worms with $5 \%$ sodium hypochlorite solution as described in Mishra et al. (2020).

\section{Determination of feeding rate}

Feeding rate (pharyngeal pumping; FR) is the rhythmic contraction of pharynx, a neuromuscular organ, which helps in the feeding of worms (Trojanowski et al. 2016). Feeding rate is pumping or movement of pharynx per unit time in the presence of food. To examine the FR, synchronized L4 staged worms were exposed with different concentrations of DDVP for $2 \mathrm{~h}$ in 96 well plate at $22{ }^{\circ} \mathrm{C}$. After exposure, the content of well including the worms were transferred into $2 \mathrm{~mL}$ centrifuge tube and mixed with $1 \mathrm{~mL}$ M9 buffer (washing off excess DDVP). The contents of the tube were allowed to settle by gravity and supernatant was removed. The worms were washed with M9 buffer twice to remove the DDVP (Cole et al. 2004). The worms were transferred to the seeded NGM plate, acclimatized for $1 \mathrm{~min}$ and analyzed for pharyngeal pumping behaviour under a compound microscope (Micros, Austria).

\section{Locomotion assay}

The locomotion assay was performed by transferring the worms on unseeded plates and allowing them to acclimatize for $1 \mathrm{~min}$. A video of crawling worms (control and DDVP exposed) was captured for $3 \mathrm{~min}$ and analyzed using WormLab software (3.0.0 Version, MBF Bioscience, Williston, VT, USA) for different behavioural parameters, such as body bending, reversal frequency, omega turn, and speed (Fig. 1). The following locomotion behaviour parameters were analysed in control and DDVP exposed worms.

Head thrashing behaviour: this is also known as swimming behaviour i.e. "lateral movement" made by worms when placed in liquid media (Buckingham, Sattelle 2009). The head thrashing behaviour of control and exposed worms was evaluated manually by transferring 20 $\mu \mathrm{L}$ of worm wash (in M9 media) onto a slide and examining under the compound microscope.

Speed analysis: speed of a worm was measured as the distance covered by the worm per unit time in a forward direction.

Body bending: this behaviour is one of the early biomarkers used for toxicological studies involving defects in motor neurons and ultimately loss of motor functions due to the exposure of toxicants, such as pesticides, drugs, nanoparticles, etc. (Nawa, Matsuoka 2012; Nawa et al. 2012).

Reversal frequency behaviour: reversal frequency locomotion behaviour of C. elegans is exhibited in order to avoid unwanted, unavoidable chemicals, environment, toxicants etc.

Omega turn behaviour: this is a body bending behaviour of C. elegans exhibited in order to avoid toxicants (Huang et al. 2006). In this behaviour, worms fold their body to form the shape of Greek letter omega $(\Omega)$.

To analyze the above mentioned behavioural parameters, the control and DDVP-exposed worms were observed under a compound microscope (Micros, Austria) 


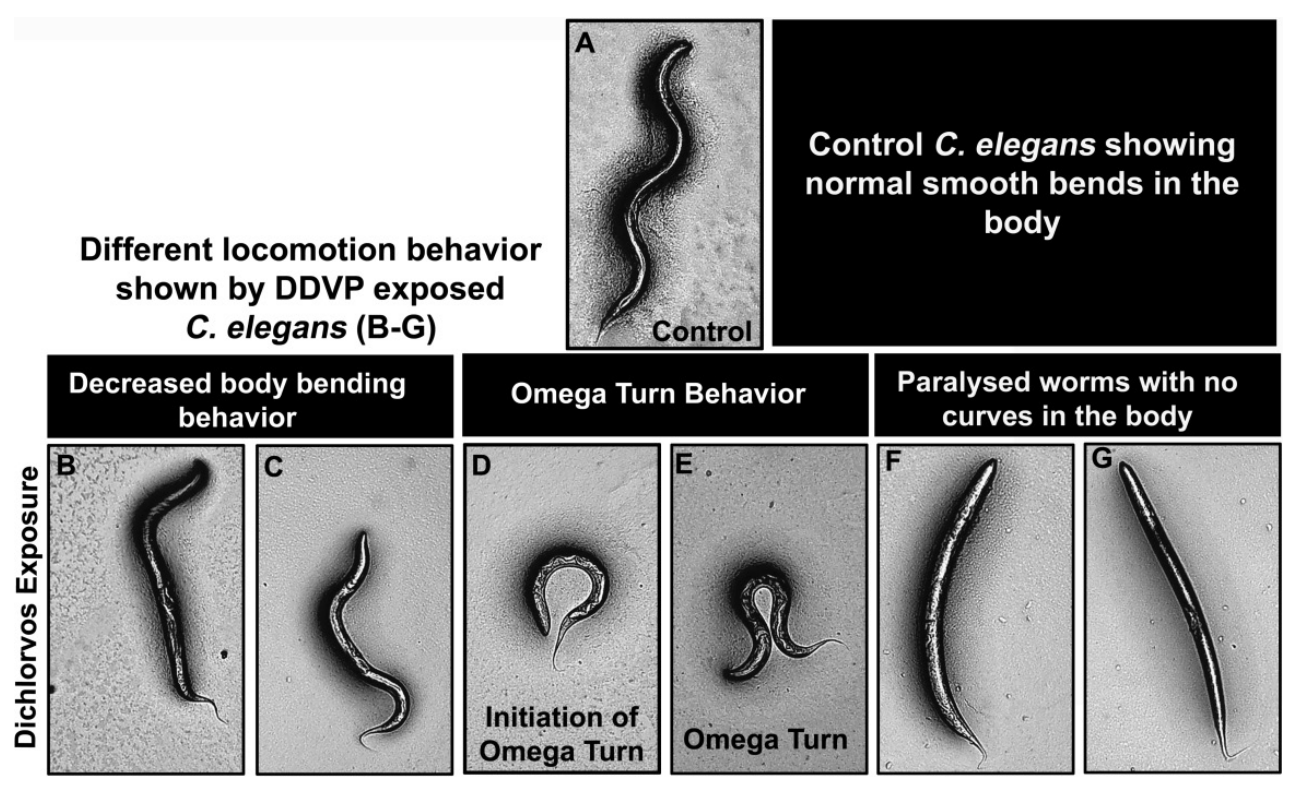

Fig. 1. Images showing the locomotion behavior in C. elegans. Unexposed C. elegans, showing normal body curvature and movement (A). C. elegans exposed with DDVP showing altered locomotion behavior such as decreased body bending (B \& C), formation of omega turn (D \& E) and almost paralyzed worm with no body bending/curves in the body (F \& G).

and analyzed using WormLab software (3.0.0 Version, $\mathrm{MBF}$ Bioscience, Williston, VT, USA).

\section{Statistical analysis}

All the data are represented as means \pm standard deviation. The biological replicates were shown as $\mathrm{n}$ and the graphs were generated using GraphPad Prism software version 5, San Diego, CA, USA. Significance between groups was analyzed by one-way ANOVA analysis with the Tukey's Test using GraphPad Prism 5.0. Images were assembled using Adobe Photoshop 7.0(R).

\section{Results}

\section{Pharyngeal pumping behaviour}

Pharyngeal pumping behaviour in DDVP exposed worms was observed to analyze the effect of DDVP exposure at increasing concentration on feeding behaviour in C. elegans. Exposure of worms to DDVP at varying concentrations for $2 \mathrm{~h}$ at $22^{\circ} \mathrm{C}$ resulted in concentration-dependent inhibition of pharyngeal pumping in C. elegans (Fig. 2B). About 15\% reduction in pharyngeal pumping behaviour in worms exposed with $5 \mu \mathrm{M}$ DDVP was observed, and 28, 35 and $80 \%$ reduction in worms exposed with 20,45 , and $70 \mu \mathrm{M}$ DDVP, respectively. Further, a complete loss of pharyngeal pumping behaviour occurred in $90 \mu \mathrm{M}$ DDVP exposed worms, suggesting that the toxicity associated with DDVP.

\section{Locomotion behaviour}

A significant concentration-dependent decrease in head thrashing behaviour $(62,77$ and $99 \%$ reduction in 5,20 and $70 \mu \mathrm{M}$ DDVP treatments, respectively) in exposed
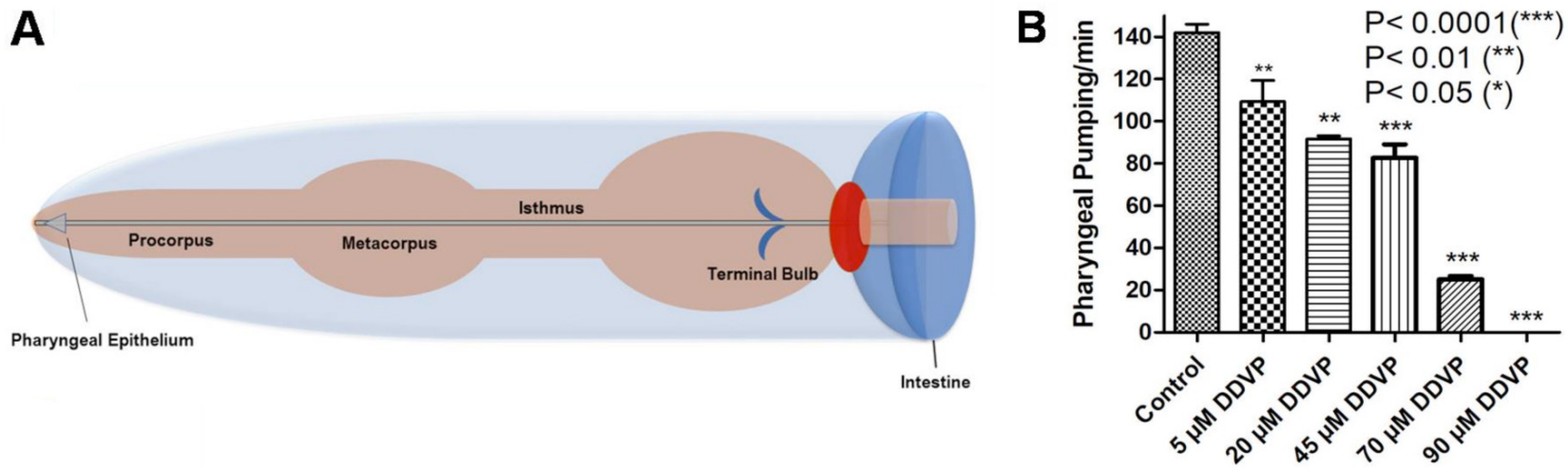

Fig. 2. A, diagrammatic representation showing different parts of pharynx in C. elegans. B. Graph showing the pharyngeal pumping behavior in $C$. elegans exposed with varying doses of DDVP for $2 \mathrm{~h}$ at $22{ }^{\circ} \mathrm{C}$. The data are represented as mean $\pm \mathrm{SD}, n=9$, one-way ANOVA: ${ }^{\star \star \star} P<0.0001,{ }^{\star \star} P<0.01,{ }^{\star} P<0.05$ represent significant difference between control and exposed groups. 


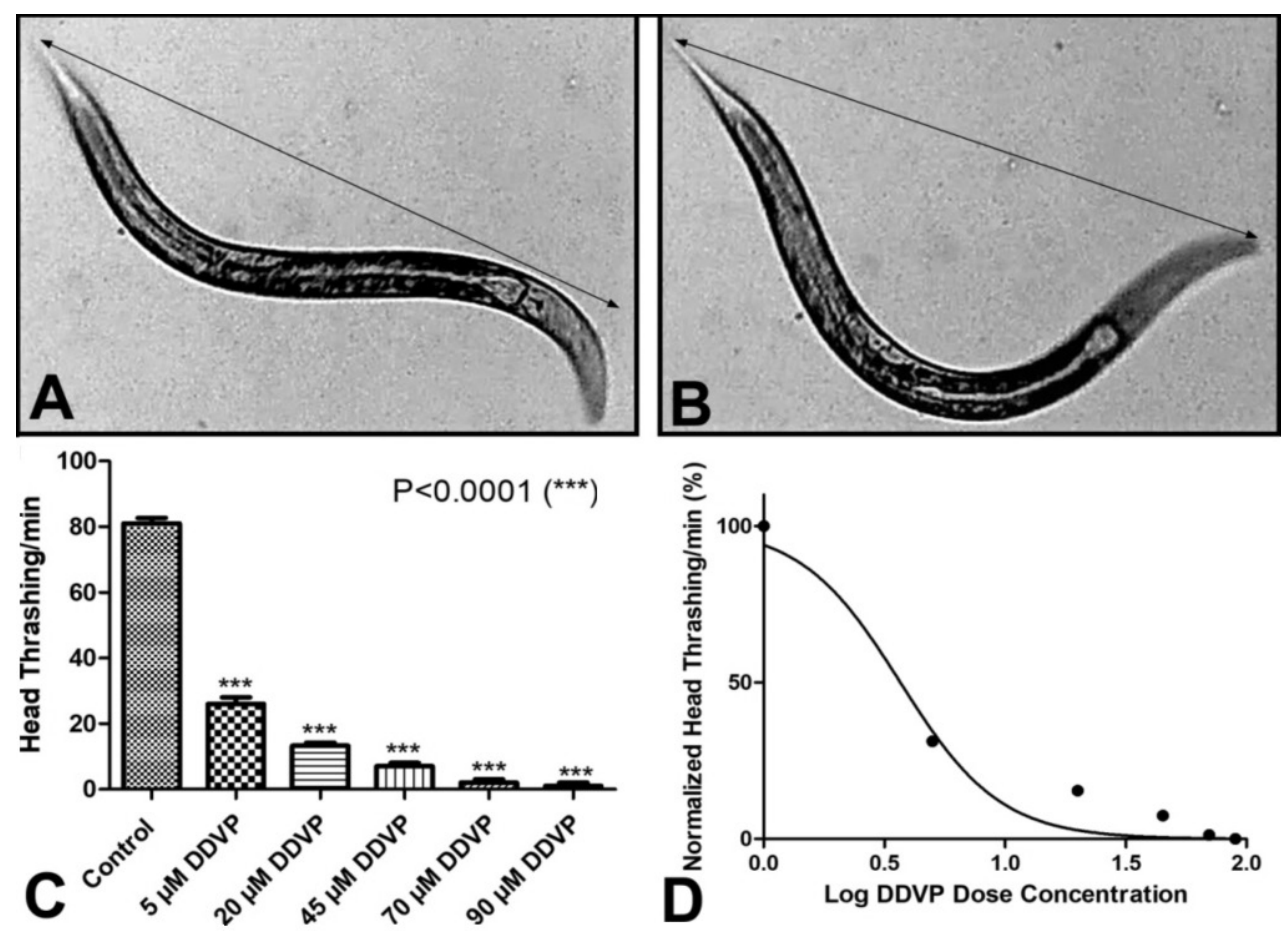

Fig. 3. A and B, one head thrash event in C. elegans. When C. elegans changes its body posture from A to B, it counts for one head thrash. $\mathrm{C}$, graph showing head thrashing behavior in C. elegans exposed to DDVP for $2 \mathrm{~h}$ at $22^{\circ} \mathrm{C}$. The data are represented as mean $\pm \mathrm{SD}, n=$ 9, one-way ANOVA: ${ }^{* * *} P<0.0001$ represent significant difference between control and exposed groups. D, dose response curve of the head thrashing behavior in C. elegans exposed to DDVP for $2 \mathrm{~h}$ at $22^{\circ} \mathrm{C}$.
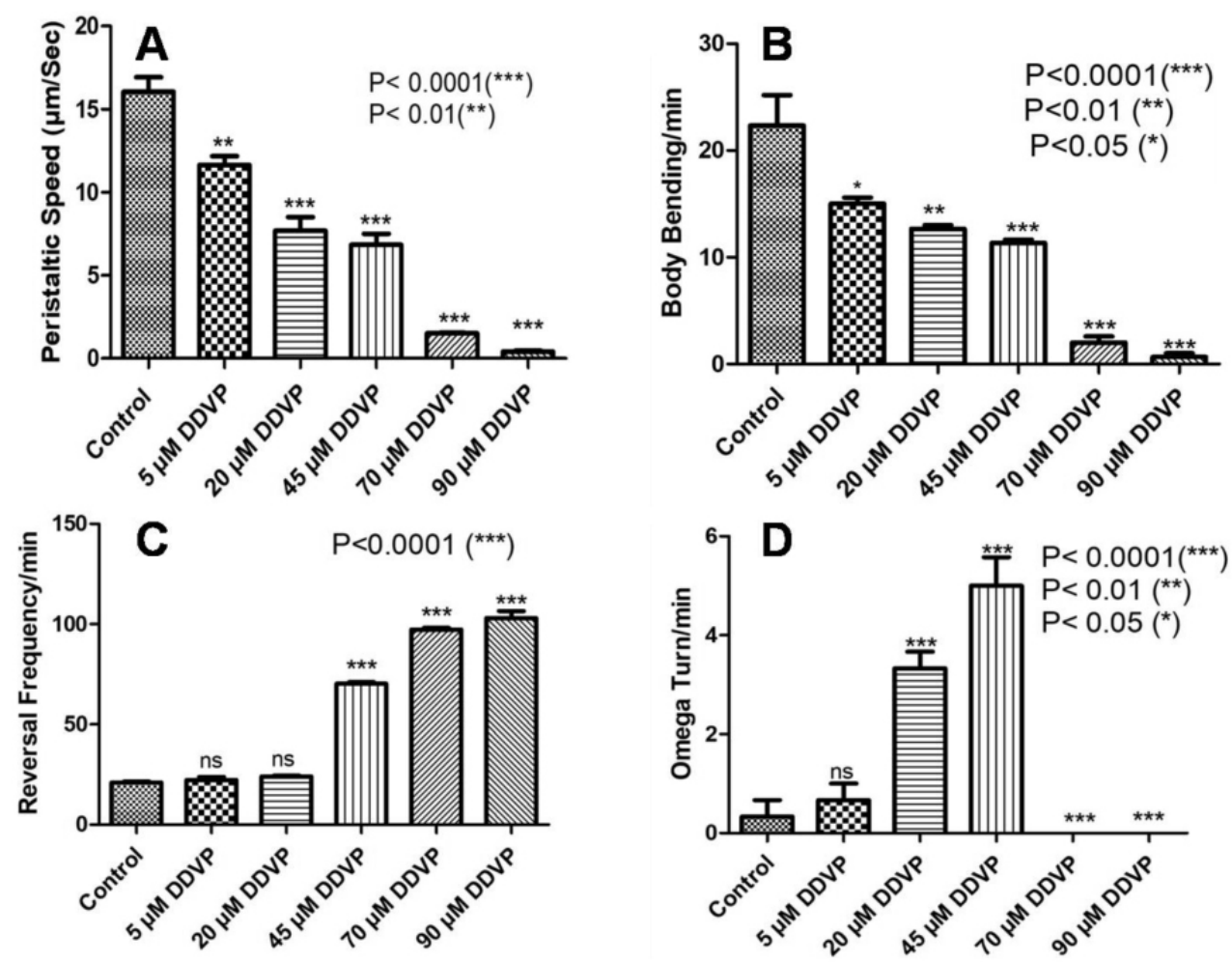

Fig. 4. The effect of DDVP exposure on locomotion behavior in C. elegans. Effect of peristaltic speed (A), body bending (B), reversal frequency (C) and omega bend locomotion (D) in control and DDVP exposed worms. The data are represented as mean \pm SD, $n=9$, one-way ANOVA: ${ }^{\star \star \star} P<0.0001,{ }^{\star \star} P<0.01,{ }^{\star} P<0.05$ represent significant difference between control and exposed groups. 
C. elegans was observed (Fig. 3 C \& D). Speed analysis in DDVP exposed worms ( $5 \mu \mathrm{M}$ to $90 \mu \mathrm{M}$ ) showed 27 to $97 \%$ reduction in speed (Fig. 4A).

The body bending analysis of age synchronized L4 stage C. elegans, exposed to 5, 20, 45, 70, and $90 \mu \mathrm{M}$ DDVP showed a concentration-dependent decrease in body bending behaviour of the worms, with $6,25,33,88$ and $96 \%$ reduction, respectively (Fig. 4B). The reversal frequency behaviour of DDVP exposed worms was also examined. A significant increase in reversal frequency behaviour of DDVP exposed worms with increasing concentrations of DDVP exposure was evident (Fig. 4C).

It was also noted that DDVP exposure to $C$. elegans increases toxicant avoidance behaviour in C. elegans. This was done by observing the formation of a omega $(\Omega)$ shape in DDVP exposed worms. It was observed that exposure of DDVP at different doses, at $22^{\circ} \mathrm{C}$ for $2 \mathrm{~h}$, resulted in altered omega turn behaviour in synchronized L4 C. elegans (Fig. 4D). Omega turn behaviour increased with increase of DDVP dose till $45 \mu \mathrm{M}$ DDVP, while worms exposed to 70 and $90 \mu \mathrm{M}$ DDVP were almost paralyzed and intensity of omega turn behaviour diminished.

\section{Discussion}

The use of pesticides has significantly increased to fulfill the food and other demands of the ever growing global population. This also raises several health concerns to human and other living organisms of various ecosystems. Thus, there is an urgent need to characterize and investigate the toxic effects of each and every pesticide. One of important points in this respect is to find new early biomarkers of toxicity to examine the developmental, behavioural and neuronal aspects associated with various environmental toxicants, including pesticides.

In the current study, we demonstrated that exposure of C. elegans to increasing concentration of DDVP resulted in dose-dependent alteration in feeding behaviour in the exposed worms. The altered pharyngeal pumping behaviour observed in DDVP exposed worms might be due to the effect of DDVP exposure on food avoidance behaviour and the toxic nature of DDVP. This is an accordance of previous studies by Jadhav and Rajini (2009) and Kisan et al. (2015).

Analysis of alteration in locomotion behaviour in worms and other animal models under the influence of toxicant is the key to assess the toxicity and its impact on animal behaviour. Locomotion in C. elegans is initiated with coordination of dorsal and ventral muscles and release of acetylcholine, a neurotransmitter that leads to propagation of a sinusoidal wave in forward (head to tail) and reverse (tail to head) directions (Zhen, Samuel 2015). When these sinusoidal waves propagate from head to tail, the worm moves in forward direction, while when it is propagated from tail to head, the worm exhibits reversal frequency locomotion behaviour (Maulik et al. 2017). Locomotion behaviour was examined by observing several end points, such as head thrashing, speed, body bend, reversal frequency, and omega turn, in DDVP exposed C. elegans.

The locomotion analysis of DDVP-exposed worms showed a significant decrease in head thrashing behaviour, decrease in speed and body bending, increased reversal frequency, and omega turn behaviour of the worm. This suggested that exposure of DDVP resulted in behavioural toxicity, due to its negative impact on motor and neuronal functions. This is in accordance with several previous studies suggesting the adverse impact of toxicant exposure on feeding behaviour in animals, which subsequently resulted in several phenotypic anomalies, such as hampered or reduced growth/development and altered behavior, including locomotion (Sandheinrich, Atchison 1990; Rao 2014; Dubinska-Magiera et al. 2016; Wexler et al. 2016; Wang 2019). Thus, the dose-dependent alteration in feeding and locomotion behaviour seen in DDVP exposed worms in the present study suggested the adverse effects of DDVP exposure on neuronal/motor function.

\section{Conclusions}

The present study suggested that DDVP poses behavioural toxicity in exposed C. elegans. It is recommended that the studied feeding and locomotion behavioural parameters can be used as early biomarkers for the study of behavioural toxicity associated with various environmental chemicals/ toxicants and in initial screening of these.

\section{Acknowledgements}

Authors acknowledge The Vice Chancellor, National Forensic Sciences University (NFSU) and Dean, School of Forensic Science, NFSU, Gandhinagar, Gujarat, India for providing infrastructure support and other facilities for our research work. Financial assistance from Scheme of Developing High Quality Research (SHODH) to SM is duly acknowledged. The authors declare that there is no conflict of interest.

\section{References}

Aktar M.W., Sengupta D., Chowdhury A. 2009. Impact of pesticides use in agriculture: their benefits and hazards. Interdisc. Toxicol. 2: 1-12.

Apfeld J., Alper S. 2019. What can we learn about human disease from the nematode C. elegans? Methods Mol. Biol. 1706: 53-75.

Brenner S. 1974. The genetics of Caenorhabditis elegans. Genetics 77: 71-94.

Buckingham S.D., Sattelle D. B.2009. Fast, automated measurement of nematode swimming (thrashing) without morphometry. BMC Neurosci. 10: 84.

Cole R.D., Anderson G.L., Williams P.L. 2004. The nematode Caenorhabditis elegans as a model of organophosphateinduced mammalian neurotoxicity. Toxicol. Appl. Pharmacol. 194: 248-256.

Dubinska-Magiera M., Daczewska M., Lewicka A., MigockaPatrzałek M., Niedbalska-Tarnowska J., Jagla K. 2016. 
Zebrafish: a model for the study of toxicants affecting muscle development and function. Int. J. Mol. Sci. 17: 1941.

Gill H.K., Garg H. 2014. Pesticides: environmental impacts and management-strategies. In: Larramendy M.L., Soloneski S. (eds) Pesticides - Toxic Aspects. Intech, Rijeka, pp. 187-230.

Gupta R.C., Sachana M., Mukherjee I.M., Doss R.B. 2018. Organophosphates and carbamates. In: Gupta R.C. (ed) Veterinary Toxicology. $3^{\text {rd }}$ Ed. Academic Press, London, pp. 495-508.

Gupta R.C. 2006. Classification and uses of organophosphates and carbamates. In: Gupta R.C.(ed) Toxicology of Organophosphate \& Carbamate Compounds. Academic Press, London, pp. 5-24.

Hart A.C. 2006. Behavior. In: The C. elegans Research Community (eds) WormBook. http://www.wormbook. org.

Hobert O. 2013. The neuronal genome of Caenorhabditis elegans. In: The C. elegans Research Community (eds) WormBook. http://www.wormbook. org.

Huang K., Cosman P., Schafer W.R. 2006. Machine vision based detection of omega bends and reversals in C. elegans. J. Neurosci. Methods 158: 323-336.

Hunt P. R. 2017. The C. elegans model in toxicity testing. J. Appl. Toxicol. 37: 50-59.

Jadhav K.B., Rajini P.S. 2009. Neurophysiological alterations in Caenorhabditis elegans exposed to dichlorvos, an organophosphorus insecticide. Pestic. Biochem. Physiol. 94: 79-85.

Jadhav K.B., Rajini P.S. 2009. Evaluation of sublethal effects of dichlorvos upon Caenorhabditis elegans based on a set of end points of toxicity. J. Biochem. Mol. Toxicol. 23: 9-17.

Kinser H.E., Pincus Z. 2018. High-throughput screening in the $C$. elegans nervous system. Mol. Cell. Neurosci. 80: 192-197.

Kisan B., Rajini P.S., Shivaya S., Shruthi N.K. 2015. Elucidation of ageing on low dose exposure to neurotoxicant dichlorvos in invertebrate model Caenorhabditis elegans. MOJ Cell. Rep. 2: 129-134.

Kisan B., Shruthi N.K., Shivaya S., Rajini P.S., Mahendra Kumar H. 2018. Dichlorvos induced toxicity and oxidative stress in the nematode Caenorhabditis elegans. J. Pharmacogn. Phytochem. 7: 2993-2996.

Levine M.J. 2007. Pesticides: a Toxic Time Bomb in Our Midst. Praeger Publishers, Westprot.

Maulik M., Mitra S., Bult-Ito A., Taylor B.E., Vayndorf E.M. 2017. Behavioral phenotyping and pathological indicators of Parkinson's disease in C. elegans models. Front. Genet. 8: 77.

Mishra S., Gaur A.V., Agarwal R. 2020. Standardization of synchronization procedure to collect the similar aged $C$. elegans. Peer Rev. J. Forens. Gen. Sci. 4: 268-271.

Nawa M., Matsuoka M. 2012. The method of the body bending assay using Caenorhabditis elegans. Bio-protocol 2: e253.

Okoroiwu H.., Iwara I.A. 2018. Dichlorvos toxicity: A public health perspective. Interdisc. Toxicol. 11: 129-137.

Owoeye O., Edem F.V., Akinyoola B.S., Rahaman S., Akang E.E., Arinola G.O. 2012. Toxicological changes in liver and lungs of rats exposed to dichlorvos before and after vitamin supplementation. Eur. J Anat. 16: 190-198.

Perni M., Casford S., Aprile F.A., Nollen E.A., Knowles T.P.J., Vendruscolo M., Dobson C.M. 2018. Automated behavioral analysis of large C. elegans populations using a wide field-ofview tracking platform. JoVE J. 141.

Raley-Susman K.M., Chou E., Lemoine H. 2018. Use of the model organism Caenorhabditis elegans to elucidate neurotoxic and behavioral effects of commercial fungicides. In: McDuffie (ed)
Neurotoxins. Intech Open, London, pp. 37-54.

Rao J.V. 2014. Locomotor behavioral response of mosquitofish (Gambusia affinis) to subacute mercury stress monitored by video tracking system. Drug Chem. Toxicol. 30: 383-397.

Rao J.C.S., Neelima P., Rao K.G. 2017. A review on the toxicity and other effects of dichlorvos, an organophosphate pesticide to the freshwater fish. Biosci. Discov. 8: 402-415.

Reilly L.P.O., Luke C.J., Perlmutter D.H., Silverman G.A., Pak S.C. 2015. C. elegans in high-throughput drug discovery. Adv. Drug Deliv. Rev. 69-70: 247-253.

Ruszkiewicz J.A., Pinkas A., Miah M.R., Weitz R.L., Lawes M., Akinyemi A.J., Ijomone O.M., Aschner M. 2018. C. elegans as a model in developmental neurotoxicology. Toxicol. Appl. Pharmacol. 354: 126-135.

Sandheinrich M.B., Atchison G.J. 1990. Sublethal toxicant effects on fish foraging behavior: Empirical vs. mechanistic approaches. Environ. Toxicol. Chem. 9: 107-119

Schwendeman A.R., Shaham S. 2016. A high-throughput small molecule screen for C. elegans linker cell death inhibitors, PLOS ONE 11: e0164595.

Shukla S., Jhamtani R.C., Dahiya M.S., Agarwal R. 2017. Oxidative injury caused by individual and combined exposure of neonicotinoid, organophosphate and herbicide in zebra fish. Toxicol. Rep. 4: 240-244.

Sisman T. 2010. Dichlorvos-induced developmental toxicity in zebrafish. Toxicol. Industr. Health. 26: 567-573.

Stiernagle T. 2006. Maintenance of C. elegans. In: The C. elegans Research Community (eds) WormBook. http://www. wormbook. org.

Taylor P., Rajini P.S., Melstrom P., Williams P.L. 2008. A comparative study on the relationship between various toxicological endpoints in Caenorhabditis elegans exposed to organophosphorus insecticides. J. Toxicol. Environ. Health A 71: 1043-1050.

Taylor J.T., Davis E., Dabisch P., Horsmon M., Matson K., Crouse C., Mioduszewski R. 2008. Acute toxic effects of inhaled dichlorvos vapor on respiratory mechanics and blood cholinesterase activity in guinea pigs. Inhal. Toxicol. 20: 46572.

Teo E., Soo Y.S.L., Fong S., Larbi A., Wright G.D., Tolwinski N., Gruber J. 2020. A high throughput drug screening paradigm using transgenic Caenorhabditis elegans model of Alzheimer's disease. Transl. Medic. Aging 4: 11-21.

Thorpe E., Wilson A.B., Dix K.M., Blair D. 1972.Teratological studies with Dichlorvos vapour in rabbits and rats. Arch. Toxicol. 30: 29-38.

Trojanowski N., Raizen D., Fang-Yen C. 2016. Pharyngeal pumping in Caenorhabditis elegans depends on tonic and phasic signaling from the nervous system. Sci. Rep. 6: 22940.

Ubels J.L., Edelhauser H.F., Meyer L.A., Worth C. 1987. Occular miotic and irritative effects of aerosol and topically applied dichlorvos and propoxur insecticides on rabbit and monkey eyes. J. Toxicol. Cutan. Occul. 6: 61-77.

Varò I., Navarro J.C., Amat F., Guilhermino L. 2003. Effect of dichlorvos on cholinesterase activity of the European sea bass (Dicentrarchus labrux). Pest. Biochem. Physiol. 75: 61-72.

Varò I., Navarro J.C., Nunes B., Guilhermino L. 2007. Effects of dichlorvos aquaculture treatments on selected biomarkers of gilthead sea bream (Sparus aurata L.) fingerlings. Aquaculture 266: 87-96.

Varò I., Amat F., Navarro J.C. 2008. Acute toxicity of dichlorvos to Aphanius iberus (Cuvier \& Valenciennes, 1846) and its anti- 
cholinesterase effects on this species. Aquat. Toxicol. 88: 53-61.

Wang D. 2019. Toxicity induction in neurons and muscle in nematodes exposed to environmental toxicants or stresses. In: Target Organ Toxicology in Caenorhabditis elegans. Springer, Singapore, pp.. 147-196.

Wang J., Cheng W., Ding W., Zhao Z. 2004. The effect of the insecticide dichlorvos on esterase activity extracted from the psocids, Liposcelis bostrychophila and L . entomophila. J. Insect Sci. 4: 1-5.

Weiss B., Amler S., Amler R.W. 2004. Pesticides. Pediatrics 113: 1030-1036.

Wexler P., Judson R., de Marcellus S., de Knecht J., Leinala E. 2016. Health effects of toxicants: Online knowledge support. Life
Sci. 145: 284-293.

WHO (World Health Organization). 1992. International Programe on chemical safety. WHO recommended classification of pesticide by hazards and guidelines to classification 19941995, UNEP/ILO/WHO.

Wittkowski P., Marx-Stoelting P., Violet N., Fetz V., Schwarz F., Schönfelder G., Vogl S. 2019. Caenorhabditis elegans as a promising alternative model for environmental chemical mixture effect assessment-a comparative study. Environ. Sci. Technol. 53: 12725-12733.

Zhen M., Samuel A.D.T.2015. C. elegans locomotion: small circuits, complex functions. Curr. Opin. Neurobiol. 33: 117-126. 